\title{
Nonstretching NMO correction of prestack time-migrated gathers using a matching-pursuit algorithm
}

\author{
Bo Zhang ${ }^{1}$, Kui Zhang ${ }^{2}$, Shiguang Guo ${ }^{1}$, and Kurt J. Marfurt ${ }^{1}$
}

\begin{abstract}
Wide-azimuth, long-offset surveys are becoming increasingly common in unconventional exploration plays where one of the key routine processes is maintaining data fidelity at far offsets. The conventional NMO correction that processes the data sample-by-sample results in the well-known decrease of frequency content and amplitude distortion through stretch, which lowers the seismic resolution and hinders $\lambda \rho-\mu \rho$ and amplitude variation with offset and azimuth (AVAz) analysis of the long-offset signal. To mitigate the stretch typically associated with large offsets, we use a matching-pursuit-based normal moveout correction (MPNMO) to reduce NMO-stretch effects in long-offset data. MPNMO corrects the data wavelet-by-wavelet rather than sample-by-sample, thereby avoiding stretch. We apply our technique (1) to a set of synthetic gathers and (2) as part of a residual velocity analysis workflow to a prestack time-migrated data volume acquired over the Northern Chicontepec Basin, Mexico. Test results show that MPNMO can produce relatively nonstretched events and generate higher temporal resolution prestack gathers.
\end{abstract}

\section{INTRODUCTION}

Normal-moveout (NMO) correction applied to commonmidpoint (CMP) gathers is one of the most important routine processes applied to seismic data and is a prerequisite for CMP stack and many other procedures (Shatilo and Aminzadeh, 2000). The objective of NMO correction is to resample a finite-offset trace in a CMP gather to approximate the kinematics of a zero-offset trace. The standard NMO correction causes wavelet stretching that lowers the frequency content of the corrected reflection event at far offset. This stretching will affect all subsequent processing and inversion. For example, unmuted stacked traces exhibit lower frequency content, and therefore have lower resolution and hinder the search for subtle traps (Noah, 1996). NMO stretch also affects AVO analysis by distorting the AVO gradient (Swan 1988, 1997; Ursin and Ekren, 1995).

In flat layers, only the zero-offset traces strictly represent the correct sequence of reflection coefficients (reflectivity function); other finite-offset-corrected traces contain a distortion of the vertical reflectivity function where wavelets have been stretched or even reversed. Buchholtz (1972) is one of the first authors to quantify the artifacts introduced by the NMO correction. Dunkin and Levin (1973) study the effect of stretch in frequency domain and concluded that usual NMO correction stretches the wavelet in such a way that the spectrum of the NMO-corrected wavelet is a linearly compressed version of the original spectrum. The amount of compression depends on $x$, the source-detector separation or offset, and $V\left(t_{0}\right)$, the velocity model at normal incidence two-way traveltime $t_{0}$. Barnes (1992) analyzes the correction distortion in instantaneous frequency and instantaneous power domain, and finds a timevariant frequency distortion caused by the NMO correction. Miller (1992) studies the impact of muting on the frequency content of stacked images. Owusu and Spencer (1995) analyze the VSP moveout stretch for a horizontally stratified medium. In Noah's (1996) examples, even minor changes in frequency caused by the NMO correction have a major impact on the interpretation.

As offset increases we often encounter nonhyperbolic moveout in isotropic and anisotropic media (Dix, 1955; Bolshykh, 1956; de Bazelaire, 1988; Castle, 1994; de Bazelaire and Viallix, 1994; Alkhalifah, 1997, 1998; Ursin and Stovas, 2006; Fomel and Stovas, 2010). Such long-offset data are critical for extending the accuracy of AVO and extracting more rock property information. Dynamic correction of these kinds of wide incidence angle gathers using the hyperbolic equation will introduce not only stretch, but also large time bias that appear on NMO corrected gathers as "hockey sticks".

\footnotetext{
Manuscript received by the Editor 15 December 2011; revised manuscript received 27 August 2012; published online 13 December 2012.

${ }^{1}$ The University of Oklahoma, ConocoPhillips School of Geology and Geophysics, Norman, Oklahoma, USA. E-mail: bo.zhang-1@ ou.edu; beiergo@ ou.edu; kmarfurt@ou.edu.

${ }^{2}$ China National Petroleum Company, BGP Inc., Zhuozhou, China. E-mail: zhan6005@gmail.com.

(c) 2012 Society of Exploration Geophysicists. All rights reserved.
} 
Unfortunately, flattening such hockey sticks still results in NMO stretch. Severely stretched portions of traces are typically muted out as noise, thus sacrificing the crucial information contained in long-offset data. Although estimation of such anisotropy and long offset AVO analysis and prestack impedance inversion is our primary objective, in this paper we focus on eliminating the limitations on such analysis due to wavelet stretch.

Rupert and Chun's (1975) block-move-sum (BMS) method is perhaps the first nonmuting solution to address stretch in the NMO correction. The BMS method treats data blocks which are moved as a unit with a single dynamic correction, thus eliminating trace stretching and reducing trace distortion. The drawback to this method is that it introduces wavelet replication and discontinuity between adjacent blocks at far-offset traces where the data blocks overlap. Byun and Nelan (1997) apply a time-varying filter based on a stretch coefficient analysis to the NMO-corrected traces to reduce the loss of high frequencies. Lichman (2000) presents a phase moveout method where he substituted the phase spectrum of the minimum-offset trace for the phase spectra of each finite offset trace, thereby avoiding the usual wavelet stretch. Based on the assumption that all time samples of a digital reflected wavelet at a particular offset have the same normal moveout, Shatilo and Aminzadeh (2000) propose a constant normal moveout correction strategy which applies a constant moveout for a finite time window of a seismic trace, protecting the corrected traces from stretching and distortion. The most critical factors for successful application of this method are to have an NMO-velocity accuracy better than $1 \%$ and an accurate estimate of the window length containing the reflection event. This technique may also produce some amplitude distortion in the overlapping intervals. Hicks (2001) describes a method for removing NMO stretch during stack that uses the parabolic Radon transform. He also introduced a new transform, which is a combination of Radon and spatial Fourier transforms, to remove stretch from the NMO-corrected CMP gathers. The drawbacks of this approach are discussed by Trickett (2003).

Brouwer (2002) expands on the block-move-sum technique and suggests an alternative approach based on the correction of tapered blocks of seismic data, followed by a coherence filter (Bruland and Johansen, 1994) to compensate for the specific artifacts thus introduced. Trickett (2003) develops a stretch-free stack process; the method replaces the two steps of NMO correction and stacking with a single-step inversion to zero offset. The main disadvantage of this procedure is that an NMO-corrected CMP gather that is useful for AVO analysis is never formed. Hunt et al. (2003), however, use this method to create pseudo-NMO-corrected gathers, and then identifies numerous new prospects using the stretch-free stacking process and AVO analysis.

Hilterman and Van Schuyver (2003) develop a processing and interpretation approach for wide-angle gathers, named seismic wide-angle processing, to avoid NMO stretch for a specified target horizon. This method first prestack migrates blocks of seismic data in the common-offset domain using event-based traveltime rather than sample-based traveltime tables. The traveltimes are based on a reflection ray-tracing model for each offset. The disadvantage is that only the target horizon is truly flat in the CMP gathers after the processing. Perroud and Tygel (2004) develop a quasistatic NMO shift approach, which can be obtained from the usual dynamic NMO process in a manner similar to a block-moveout process, to avoid stretch as much as possible. This method first requires performing the usual NMO velocity analysis, which estimates $t_{0}$ and $V\left(t_{0}\right)$ for each reflection event. They adjust $V\left(t_{0}\right)$ to maintain the local traveltime parallelism for each user-identified band-limited reflection event. Unfortunately, this adjustment increases the NMO stretch effect between the identified reflection events. More recently, Masoomzadeh et al. (2010) carefully study the influence of the data block size to the distortion of the signal and note that smaller block sizes introduce stretch whereas larger block sizes generate image discontinuities at the block boundaries. They propose using isomoveout curves (lines of equal moveout) in the timevelocity panel to achieve multiblock constant moveout for the selected individual events, leading to a nonstretch correction for the selected events. Nonstretch stacking is achieved by the use of a zigzag velocity function. The main drawbacks are the potential for discontinuities at the window boundaries and the need to estimate appropriate block lengths.

We introduce a strategy which reduces the NMO stretch at far offsets using a matching pursuit wavelet decomposition technology. We start by reviewing the conventional NMO correction equation, using cartoons describing the stretch problem introduced by the conventional correction. Cartoons illustrate the wavelet replication and discontinuity problem that occurs using the block-based correction strategy. Next, we present our nonstretch NMO strategy based on matching pursuit. Finally, we apply our method to a prestack time-migrated volume acquired over the Northern Chicontepec Basin, Mexico and show the improvements on the corrected gathers and final stacked section.

\section{CONVENTIONAL NMO CORRECTION AND STRETCH}

NMO correction transforms seismic traces with arbitrary offset $x$ into their zero-offset approximations using the NMO velocity (Shatilo and Aminzadeh, 2000). Assuming a layer-cake model for the NMO correction, we obtain the well-known hyperbolic traveltime equation (Dix, 1955) as a function of two-way traveltime at zero-offset $t_{0}$, offset $x$, and NMO velocity $V\left(t_{0}\right)$ :

$$
t\left(t_{0}, x\right)=\sqrt{t_{0}^{2}+\frac{x^{2}}{V^{2}\left(t_{0}\right)}}
$$

where $V\left(t_{0}\right)$ is approximated by the root-mean-square (rms) velocity for flat-layered media. The NMO correction time at offset $x$ and zero-offset time $t_{0}$ can be written as

$$
\Delta t_{\mathrm{NMO}}=t\left(t_{0}, x\right)-t_{0}=\sqrt{t_{0}^{2}+\frac{x^{2}}{V^{2}\left(t_{0}\right)}}-t_{0} .
$$

The conventional correction is implemented on a sample-by-sample basis, using different values of $\Delta t_{\mathrm{NMO}}$ for different samples having a different value of $t_{0}$ in the trace. Consequently, samples within a single wavelet will suffer different amounts of correction, thereby causing distortion.

Figure 1 illustrates the distortion of finite-offset seismic traces for a simple case of two reflection events, R1 and R2. The time interval at zero offset between the two dashed lines is equal to the wavelet duration. We assume we know the correct NMO correction velocity through semblance-based velocity analysis. For a given reflection event, the stretching is exacerbated with increasing offset. The most severe stretching occurs at the intersection of reflection hyperbolas. 
Beyond such intersection points, the standard NMO correction gives rise to local time-reversal of the signal. These reverse waveforms are particularly harmful to stacking, high-resolution velocity analysis based on flattened events, AVO analysis, and noise suppression, as well as other techniques that benefit from the longoffset data information. Furthermore, sample values must be interpolated to fill in gaps created by the differential stretch.

\section{NONSTRETCH NMO CORRECTION}

To avoid stretching the nonzero offset traces, the moveout correction needs to be constant for all samples that belong to the same reflection wavelet. Variations of the block-based moveout correction provide a means to approximately achieve this goal.

\section{The block-based moveout NMO correction}

The block-based moveout correction has two main limitations. First, the block size needs to be a function of the time duration of the reflection events. Second, the block boundaries overlap at farther offset if the correction velocity increases with depth, giving rise to wavelet repetition and discontinuities at the block boundaries. Figure 2 illustrates the block-based correction procedure. Initially, the zero-offset trace $d_{0}(t, x=0)$ is divided into data blocks which may or may not overlap (Figure 2a). The block length $\tau$ and the block centers $t_{0}$ are the two key factors affecting the accuracy of the correction. Rupert and Chun (1975) and Shatilo and Aminzadeh (2000) suggest that the block length should be the same as the time duration of the wavelet. In the example here, the $t_{0}$ axis is subdivided into adjacent but nonoverlapping blocks. The block sizes $\tau_{1}$ and $\tau_{2}$ are set to approximate the wavelet duration. Masoomzadeh et al. (2010) modify the NMO velocity to better achieve this goal. Because the NMO velocity usually increases with depth, the traveltime of two successive blocks will be compressed at the long offset, which results in overlapping areas for adjacent blocks at far offsets, indicated by the green areas.

Each data block is corrected as a unit from the top to the bottom of the $t_{0}$ axis (Figure $2 \mathrm{~b}$ ). The samples located in the overlapping area (green) are used twice during the correction resulting in either a repetition or a discontinuity at the block boundaries. The degree of repetition worsens with increasing offset. This repetition harms the stack, creates artificial stacked reflections, and lowers the seismic resolution.

\section{The matching pursuit NMO correction}

An NMO-uncorrected trace $d(t)$ can be regarded as the convolution of the seismic wavelet with the reflectivity series and added noise:

a)

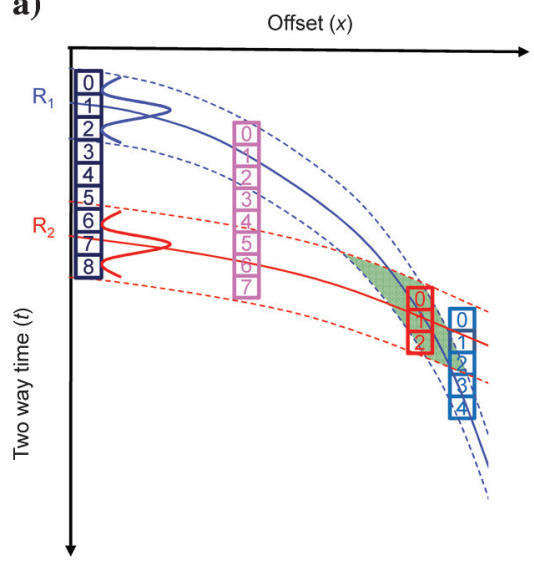

b)

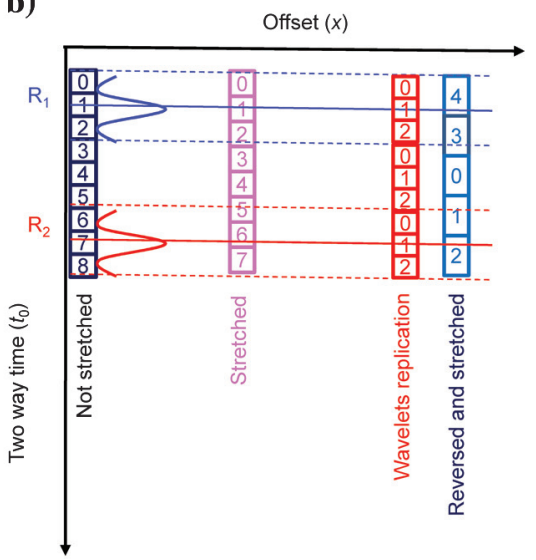

Figure 1. Schematic diagrams showing conventional NMO correction for two reflection events (R1 and R2) (a) before (b) after NMO correction. Only the zero-offset samples maintain the same waveform before and after correction; the degree of stretch increases with increasing offset. Shallower events undergo greater stretch than deeper events. The maximum stretch occurs at the crossing point, beyond which the sample's chronological order is reversed. a)

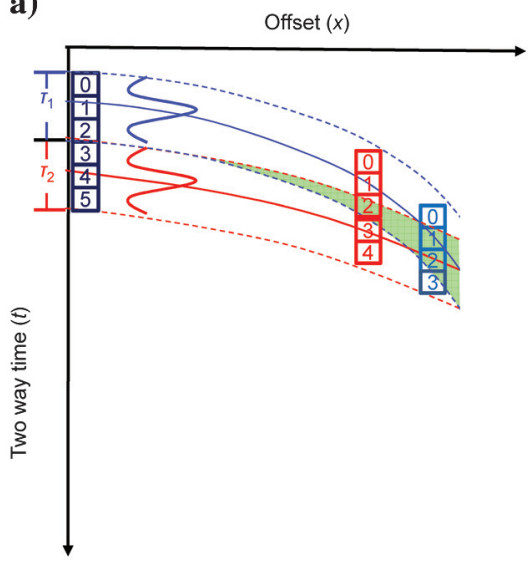

b)

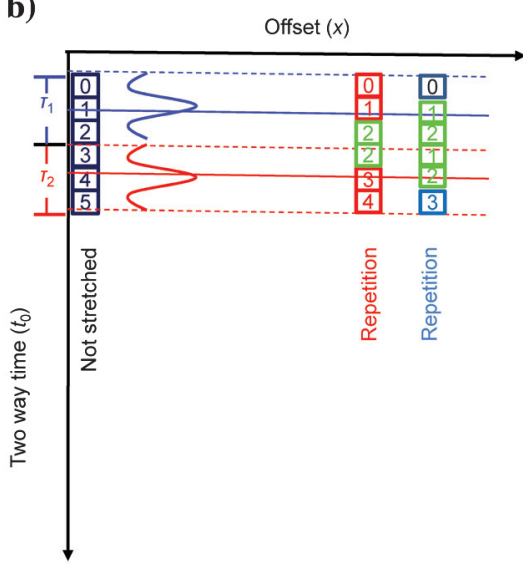

Figure 2. Cartoons illustrating the implementation and limitation of the block-based NMO correction. (a) The zero-offset time is divided into adjacent data blocks of variable time duration $\left(\tau_{1}\right.$ and $\left.\tau_{2}\right)$. The samples within each block have the same amount of moveout correction. If the NMO correction velocity increases with depth (or zero-offset traveltime), the traveltime will be compressed with increasing offset, giving rise to overlap (indicated by the green area) at the boundary between the two adjacent blocks. (b) Two interfering reflection events after block-based nonstretch NMO correction. Because of the compression of traveltime with depth, the samples indicated by green amplitude values located in the overlapping area of adjacent block will be used twice, giving rise to wavelet repetition and discontinuities. 
Our input data consist of prestack-time-migrated common-reflection-point gathers $d\left(t, x_{n}\right)$ that have been subjected to a reverse NMO correction using the migration velocity function, where $x_{n}$ is the offset of the $n$th trace in the current gather. Our output data consist of MPNMO-corrected gathers, $d_{\mathrm{MPNMO}}\left(t_{0}, x_{n}\right)$, modeled uncorrected gathers, $d_{\text {mod }}\left(t, x_{n}\right)$, and the residual or difference, $d_{\text {res }}\left(t, x_{n}\right)$, between the original and modeled uncorrected gathers. The process begins by selecting the reflection traveltime equation using either hyperbolic (equation 2) or nonhyperbolic moveout (e.g., Alkhalifah, 1997), as appropriate. Then, we build a library of analytic Ricker or Morlet wavelets. Before the MPNMO correction loops begin, residual data are initialized to the input uncorrected data, and modeled data and MPNMO-corrected data are initialized to zero. At each decomposition and correction iteration $j$, we apply a constant normal-moveout correction to the residual uncorrected data and stack the corrected gather to form a residual stacked trace. We compute its envelope $e\left(t_{0}\right)$ and pick $t_{0}^{(k)}$ of the $K$ largest envelope peaks that exceed $50 \%$ of the value of the largest peak. Then, we compute moveout times $t_{n}^{(k)}$ for each offset $x_{n}$. For each residual trace $d_{\text {res }}\left(t, x_{n}\right)$ in the current gather, we also compute its Hilbert transform to form an analytic trace and calculate the instantaneous frequency $f_{n}^{(k)}$ at time locations of $t_{n}^{(k)}$ and look up a precomputed analytic wavelet, $w\left[t, f_{n}^{(k)}\right]$. Finally, the analytic wavelets are least-squares fit to the analytic residual trace $d_{n}^{(j)}$ to obtain the amplitude, $a_{n}\left[t^{(k)}\right]$, and phase, $\varphi_{n}\left[t^{(k)}\right]$, of the analytic wavelet

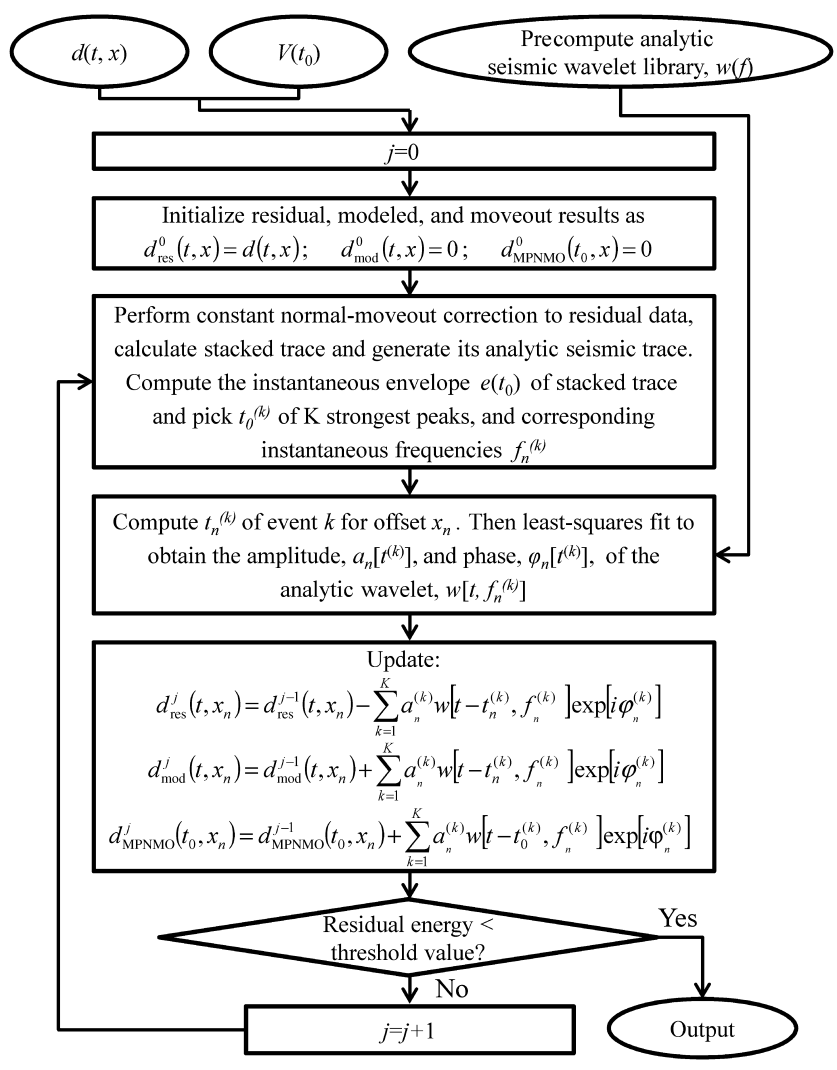

Figure 3. Flowchart showing the nonstretch NMO correction workflow based on the matching-pursuit wavelet decomposition technique. Instead of sample-by-sample, the correction is implemented on a wavelet-by-wavelet basis.
(Liu and Marfurt, 2007). The $K$ scaled wavelets are then subtracted from the previous $(j-1)$ th version of the uncorrected residual trace:

$$
\begin{aligned}
d_{\mathrm{res}}^{(j)}\left(t, x_{n}\right)= & d^{(j-1)}\left(t, x_{n}\right) \\
& -\sum_{k=1}^{K} a_{n}^{(k)} w\left[t-t_{n}^{(k)}, f^{(k)}\right] \exp \left[i \varphi_{n}^{(k)}\right],
\end{aligned}
$$

and added to the previous version of the MPNMO-corrected and modeled uncorrected traces:

$$
\begin{aligned}
d_{\mathrm{MPNMO}}^{(j)}\left(t_{0}, x_{n}\right)= & d_{\mathrm{MPNMO}}^{(j-1)}\left(t_{0}, x_{n}\right) \\
& +\sum_{k=1}^{K} a_{n}^{(k)} w\left[t-t_{0}^{(k)}, f^{(k)}\right] \exp \left[i \varphi_{n}^{(k)}\right]
\end{aligned}
$$

and

$$
\begin{aligned}
d_{\bmod }^{(j)}\left(t, x_{n}\right)= & d_{\bmod }^{(j-1)}\left(t, x_{n}\right) \\
& +\sum_{k=1}^{K} a_{n}^{(k)} w\left[t-t_{n}^{(k)}, f^{(k)}\right] \exp \left[i \varphi_{n}^{(k)}\right] .
\end{aligned}
$$

The above process is repeated until the total energy of the residual trace falls below a desired threshold (Figure 3). If there are crossing events in the gathers, the scaled wavelets are added to the time $t_{0}^{(k)}$ which has the largest stacking power.

In this paper, we assume that the MPNMO correction velocity function comes from high-resolution velocity analysis, such as the method proposed by Key and Smithson (1990). To obtain good-quality corrected gathers, the error of NMO-velocity should be less than $1 \%$, such as that for the method proposed by Shatilo and Aminzadeh (2000). Larger errors will place the wavelets at an incorrect time value of $t_{0}$, which will harm subsequent AVO and prestack-inversion processes.

To demonstrate the results that MPNMO produces, we apply the workflow shown in Figure 3 to a synthetic case. The gather is composed of three reflection events. The first and second events cross each other at middle offset (Figure $4 \mathrm{a}$ ). The offsets range from 50 to $3000 \mathrm{~m}$ at increments of $50 \mathrm{~m}$. A Ricker wavelet with a $30-\mathrm{Hz}$ dominant frequency is used to generate the synthetic gathers.

Figure $4 \mathrm{~b}$ shows the corrected results using the conventional NMO algorithm. All the events are stretched to some extent at the farthest offset, especially those events located in the rectangle. Crossing traveltime curves give rise to wavelet repetitions, indicated by circles in Figure 4b. In contrast, the MPNMO algorithm preserves wavelets during the correction (Figure 4c). Events within the rectangle in Figure 4c have anonymously low energy compared with other offsets for the second reflection. This artifact arises because MPNMO simply moves all matched wavelets at crossing time $t$ (labeled with rectangle in Figure 4a) to time $t_{0}$ of the first reflection (labeled with the rectangle in Figure $4 \mathrm{c}$ ).

To quantify the improvement of MPNMO over conventional $\mathrm{NMO}$, we first compare the spectra of the corrected reflection events for five different offsets. For conventional NMO, the spectra of the first and second reflection events shift toward lower frequencies when the offset exceeds the crossover point (Figure $5 \mathrm{a}$ and $5 \mathrm{~b}$ ). The spectral shift to lower frequencies with increasing offset is more 
a)

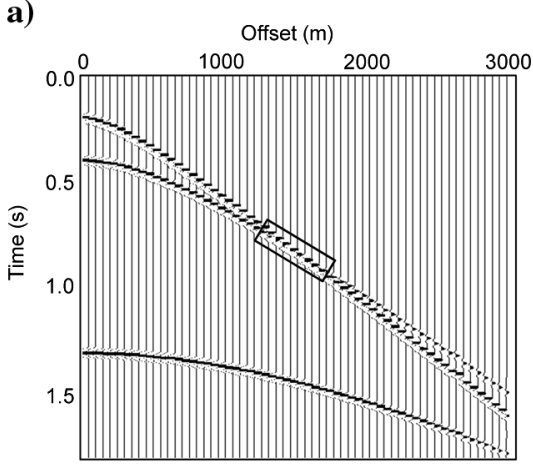

b)

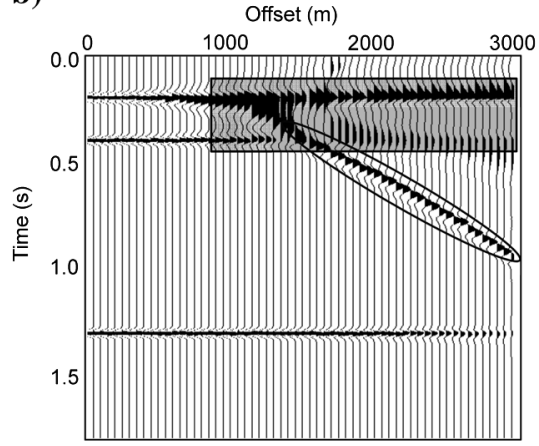

Figure 4. (a) A synthetic input gather and corresponding corrected gathers after (b) conventional NMO and (c) MPNMO corrections.

c)

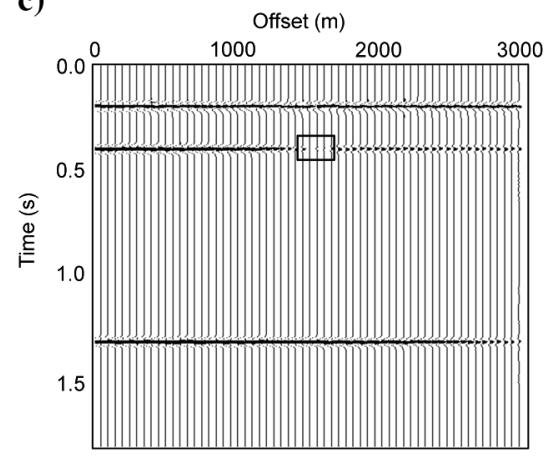

a)

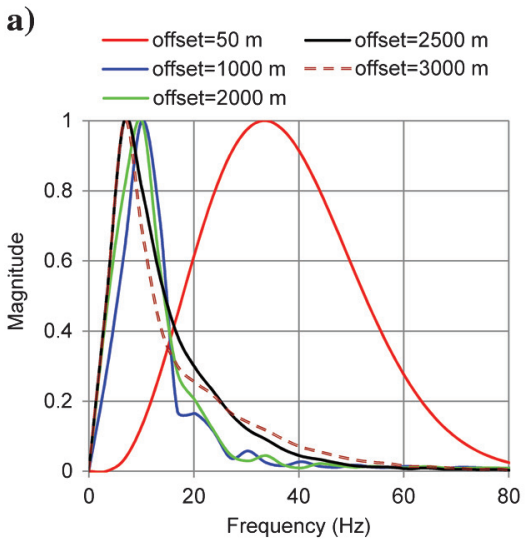

b)
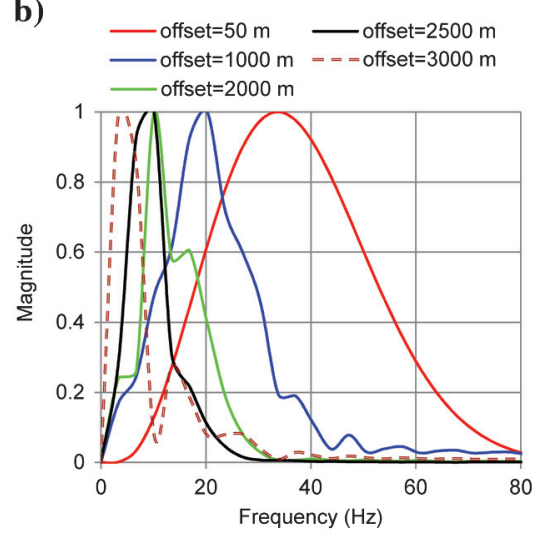

Figure 5. Representative spectra as a function of offset for conventional NMO corrected gathers for the (a) first, (b) second, and (c) third corrected reflection events shown in Figure $4 b$.

c)

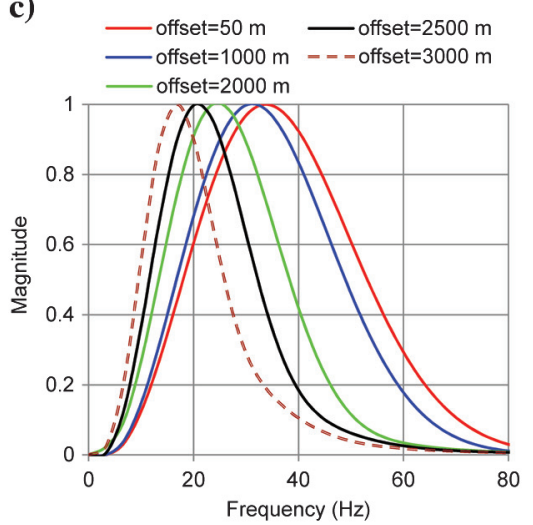


Figure 6. Representative spectra as a function of offset for MPNMO corrected gathers for the (a) first, (b) second, and (c) third corrected reflection events shown in Figure $4 \mathrm{~b}$.

Figure 7. Crosscorrelation coefficients between the zero-offset trace and finite-offset corrected traces using (a) conventional NMO and (b) MPNMO applied to the gather shown Figure $4 \mathrm{a}$.

Figure 8. A synthetic gather with random noise (a) before and (b) after MPNMO correction.

a)

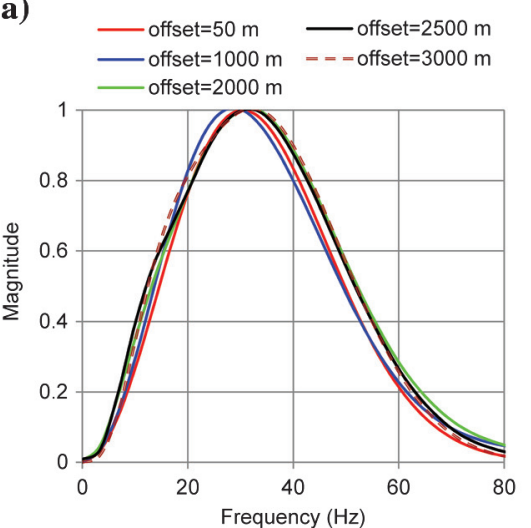

b)

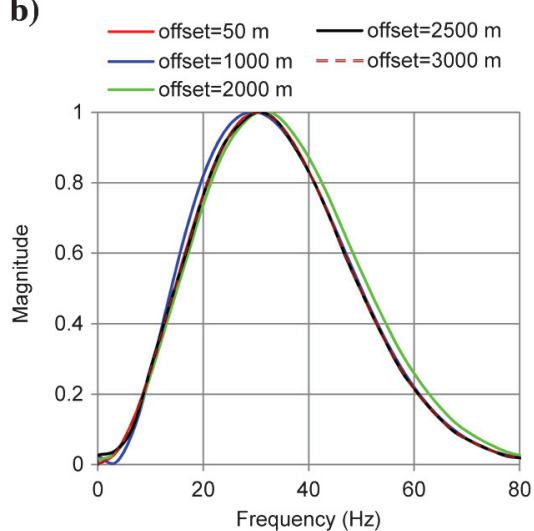

c)

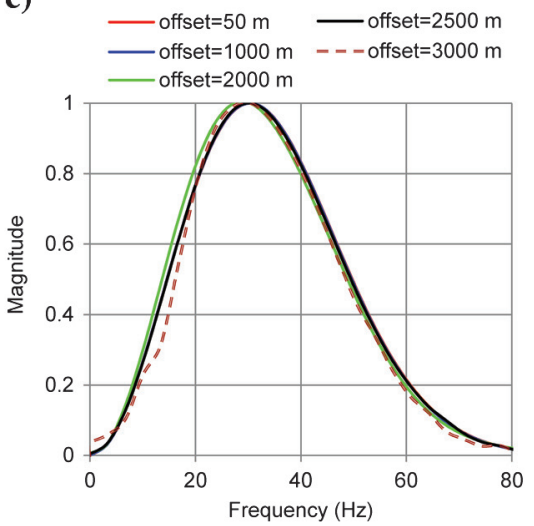

a)

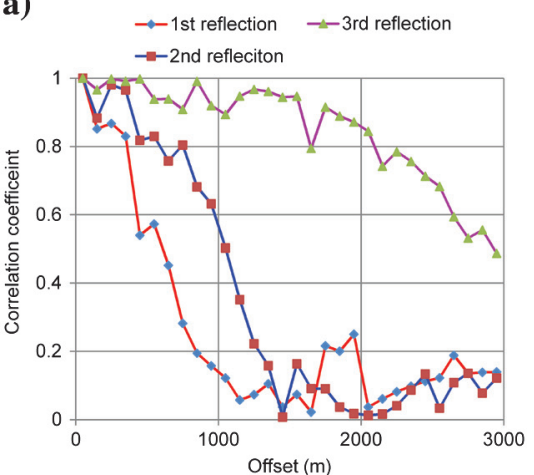

a)

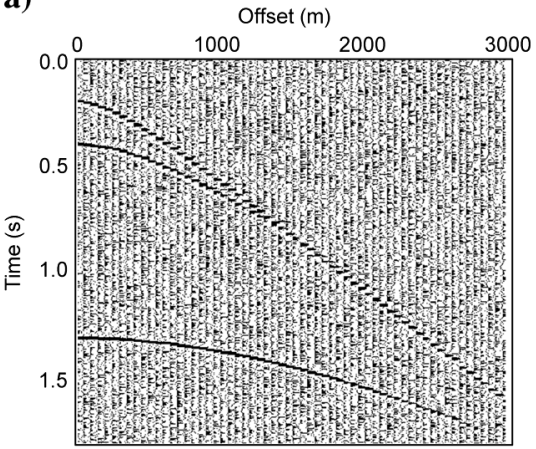

b)

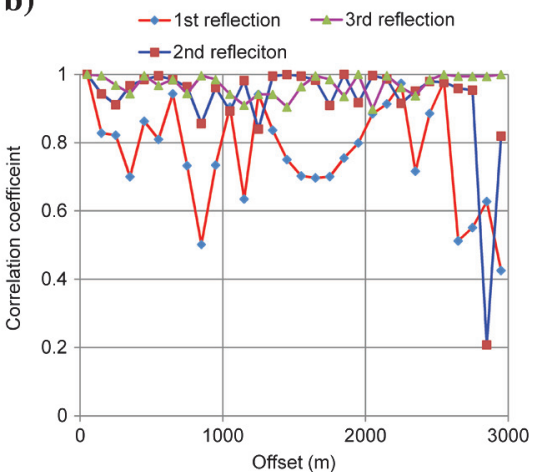

b)

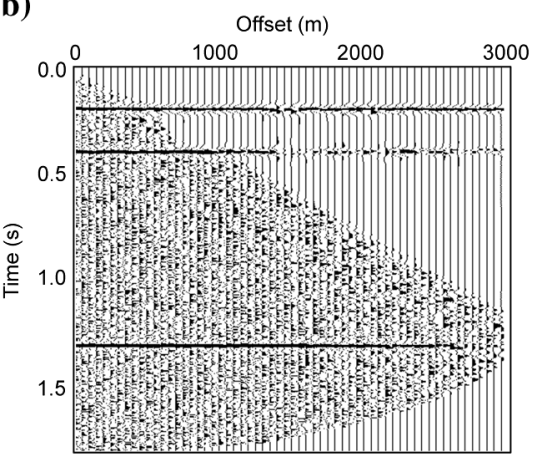


continuous for the third reflection events, but results in stretch at far offsets (Figure 5c). Figure 6 shows that the spectra of these three reflection events are much better preserved using MPNMO, with the spectra for reflectors 1-3 (Figure 6a-6c) preserved with increasing offset.

A second measure of the fidelity of the correction is the crosscorrelation coefficient between the corrected trace at small offset with the corrected traces at all other offsets, much as is done in residual statics computations (Figure 7). The MPNMO-corrected traces (Figure 7b) are much better correlated to the zero-offset trace than when using conventional NMO (Figure 7a). This waveform consistency is critical to robust $\mathrm{AVO}$ and prestack inversion results.

Figure 8 illustrates the sensitivity of MPNMO to noise. Figure 8a shows the result of adding $15 \%$ random noise to the synthetic gathers shown in Figure 4a. The noise makes it hard to see the second reflection events. The last three traces of the third reflection events are also overwhelmed by noise. Figure $8 \mathrm{~b}$ shows that MPNMO successfully corrects the noisy gathers without generating obvious artifacts. Figure 9 shows the robustness of MPNMO to velocity errors. We set the velocity for the third event to be $10 \%$ slow, resulting in an overcorrected, but relatively nonstretched, event.

Figure 10 shows the effect of the MPNMO correction on multiple suppression. Our synthetic contains two primary events (the first and third events). The second event is a multiple of the first event (Figure 10a). Figure 10b and 10c shows, respectively, the conventional NMO-corrected with $150 \%$ stretch mute and the MPNMOcorrected gathers. The multiples are not flattened, removed, and stretched in the MPNMO correction procedure, and the amount of moveout of each event is determined by the primary velocity at the corresponding time and offset. Figure 10d shows the stacked traces, where the amplitude is, respectively, scaled by the magnitude of the first corresponding reflection event. The red trace is generated from the MPNMO-corrected gather whereas the blue trace is produced from the conventional NMO-corrected gather. Due to the application of stretch mute in conventional NMO, MPNMO make the suppression of multiples easier (exhibiting lower multiple energy) in the stacked trace. The goal of this paper is to move out the data using the correct velocity, not remove multiples. However, we anticipate that we can use wavelet-based matching pursuit concepts to high-resolution Radon transform, which may provide improved multiple rejection.

\section{APPLICATION}

Having calibrated our algorithm on synthetic data, we now apply it to a residual velocity analysis workflow to prestack time-migrated CMP gathers in the Northern Chicontepec Basin, Mexico. The target tight sand Paleocene-Eocene Chicontepec formation lies between $t_{0}=0.8 \mathrm{~s}$ and $t_{0}=1.2 \mathrm{~s}$. Interpretation of the Chicontepec reservoirs is hampered by geologic complexity, overlying volcanics, and limited resolution (Sarkar, 2011). Figure 11a shows a representative CMP gather after reverse NMO correction which "resqueezes" the migration stretch. The shallow part of the conventional NMO-corrected results suffers severe stretch at far offsets. This stretch can noticeably lower the temporal resolution of the stack and is harmful to prestack inversion. Usually, such severely stretched data are muted out (Figure 11b) based on a predefined muting criteria. In this example, we allow wavelets to

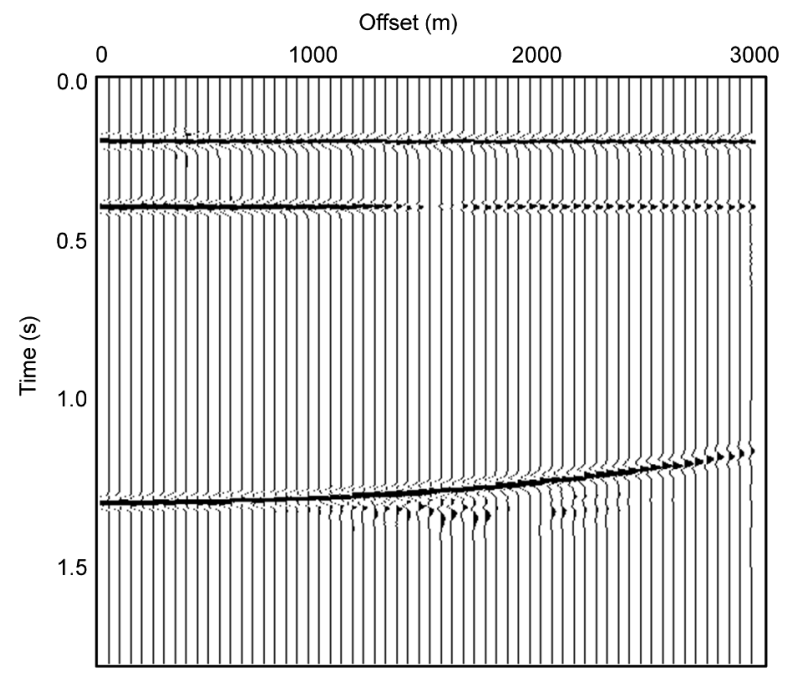

Figure 9. Although MPNMO minimizes stretch effects, it does not correct for errors in velocity. Here the gather shown in Figure 4a is corrected with MPNMO using a velocity that was $10 \%$ too slow, thereby overcorrecting the data. a)

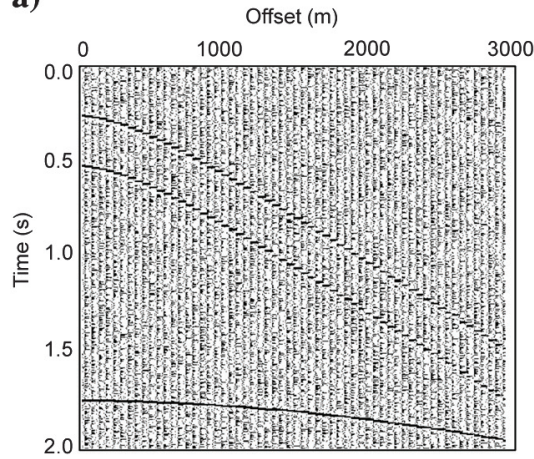

c)

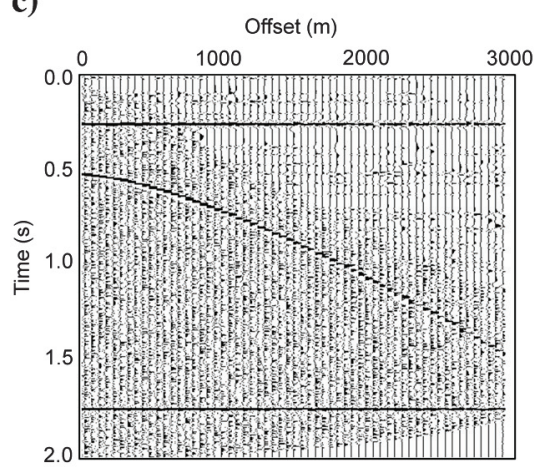

b)

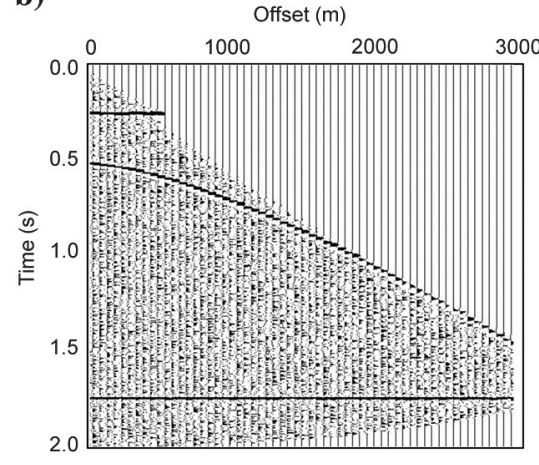

d)

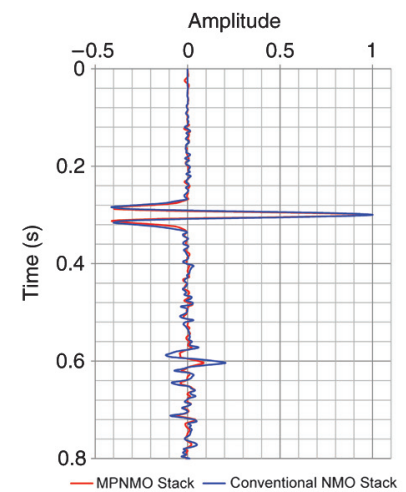

Figure 10. Stacked traces (d) after conventional NMO (b) and MPNMO (c) correction to a synthetic gather with multiples (a). 
stretch no more than $150 \%$. Next, we apply the workflow shown in Figure 3 to the same CMP gather shown in Figure 11a and obtain the MPNMO-corrected results (Figure 11c). We use a precomputed library of Morlet wavelets. MPNMO minimizes the stretch that occurs in the shallow far-offset data when compared to the conventionally NMO-corrected data (Figure 11b). These corrected far-offset data can be used to improve the stability of AVAz and $\lambda \rho-\mu \rho$ inversion.

Figure $12 \mathrm{a}$ and $12 \mathrm{~b}$ shows the amplitude spectra for angle-rangelimited stacked traces obtained from the corrected traces shown in Figure $11 \mathrm{~b}$ and $11 \mathrm{c}$. Red, blue, and green lines show the spectra of small- $\left(0^{\circ}-10^{\circ}\right)$, intermediate- $\left(10^{\circ}-20^{\circ}\right)$, and large- $\left(20^{\circ}-30^{\circ}\right)$ anglerange stacked traces. Due to the increasing stretch with the increase of incidence angle when using conventional NMO correction, the spectral bandwidth (the green line in Figure 12a) of the large-angle stacked traces is distorted and narrower than that of the small-angle stacked traces. In contrast, MPNMO preserves the spectral bandwidth for the large-angle stacks (Figure 12b).

As we did for the synthetic example, we wish to compare the change in waveform as a function of offset or angle. We show the zero-lag crosscorrelation between the small- and large-angle stacks for the shallow corrected gather in Figure 13. The time gate used for correlation is set to $50 \mathrm{~ms}$, which is the approximate time duration of one reflection event in this part. Note the overall higher correlation using MPNMO (the solid curve) versus conventional NMO (the dash curve).

After the moveout correction, we stack the corrected CMP traces such as shown in Figure $11 \mathrm{~b}$ and $11 \mathrm{c}$ to form seismic stacked sections. The traditionally corrected and stacked section (Figure 14a) is acceptable for mapping structure but not stratigraphy (Sarkar, 2011). The interfering events are not well resolved using the conventional correction, such as the pinch out located in the circles in Figure 14a and 14b. Furthermore, the reflection events are more continuous by applying MPNMO correction to the same data set; for example, the events that are labeled with the rectangle in Figure 14a and 14b. To quantify the improved resolution, we compare the average spectrum (Figure 15) of the stacked sections (Figure 14). The dash and solid curves represent the spectra of the stacked sections using conventional NMO correction (Figure 14a) and MPNMO correction (Figure 14b), respectively. The MPNMO spectrum has a greater ratio of high to low frequencies.
Figure 11. Applying (b) conventional NMO and (c) MPNMO correction to a representative prestack time migrated gather (a) from the Chicontepec Basin, Mexico (CMP no. 1 in Figure 14). a)

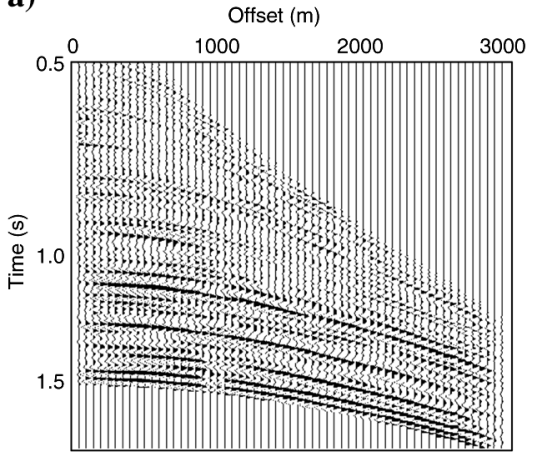

b)

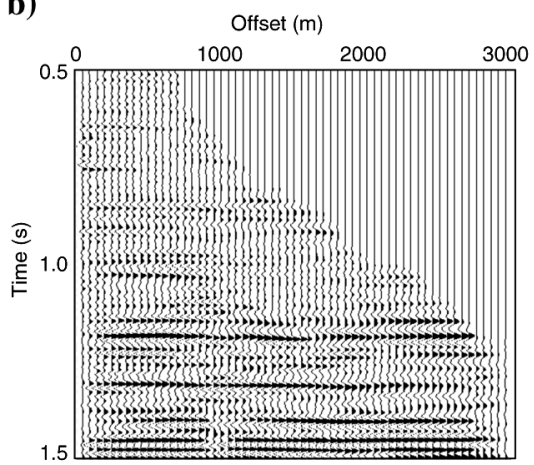

c)

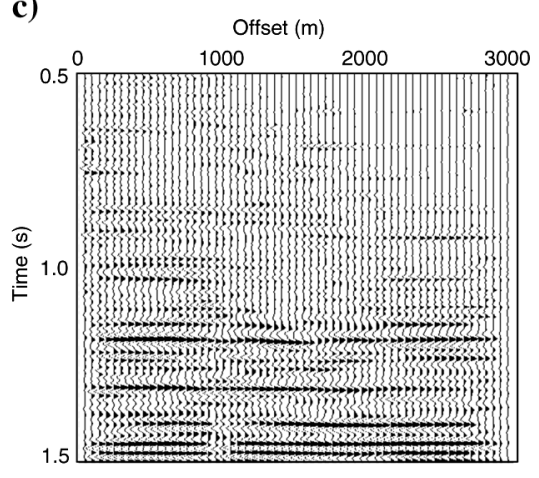

Figure 12. Spectra of small-, intermediate-, and large-angle stacks with (a) conventional NMO and (b) MPNMO correction. a)

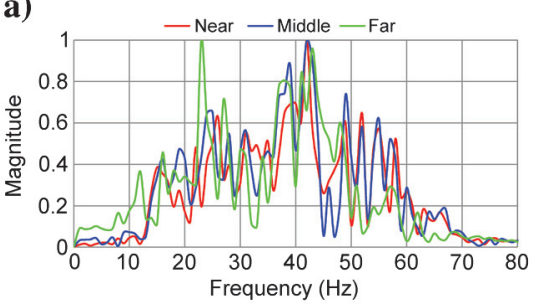

b)

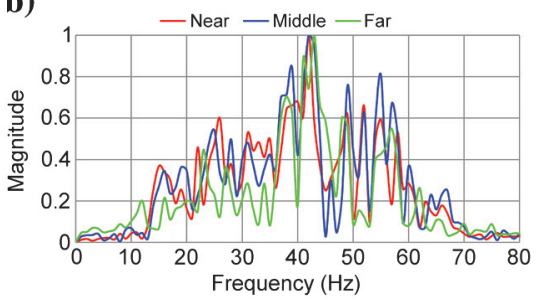




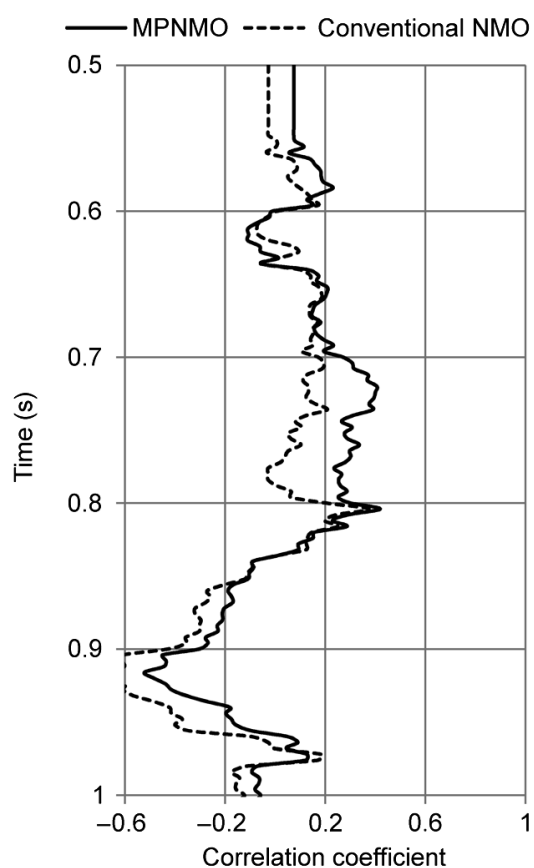

Figure 13. Correlation coefficients between the small-and large-angle stacks using conventional NMO (in dash) and MPNMO (in solid).

\section{LIMITATIONS}

Like conventional NMO, MPNMO will generate under- (or over-) corrected traces if the velocity function is higher (or lower) than it should be. Although the cost of MPNMO is some 15 to 20 times the cost of conventional NMO, and significantly greater than the cost of competing published nonstretch NMO correction algorithms, the cost is notably less than the prestack time migration algorithm used to generate the input gathers. Through parallelization and precomputation, not only of wavelets but of moveout functions, the cost becomes acceptable. Crossing events can be approximately handled but produces amplitude artifacts that could harm subsequent AVO and prestack inversion workflows. Proper partitioning of this energy to the appropriate $t_{0}$ location may require integrating MPNMO concepts with high-resolution Radon transforms.

\section{CONCLUSIONS}

Conventional NMO corrections introduce stretch at offsets that are large relative to reflection depth. This stretch lowers the seismic temporal resolution and distorts the seismic waveform. Block-based correction algorithms avoid stretch but result in wavelet repetition at the block boundary, giving rise to artifacts. Our matching pursuit NMO correction is implemented on a wavelet-by-wavelet basis, thereby reducing stretch, avoiding wavelet repetition, and improving the temporal resolution. By minimizing stretch, MPNMO improves waveform consistency for the reflection events along the offset axis. As a result, more far-offset data are available for subsequent $\lambda \rho-\mu \rho$ and AVAz inversion as well as increased leverage against multiples. The final stacked section has improved bandwidth, which is critical for interpreting thin reservoirs. We feel it is advantageous to apply our algorithm to the prestack time migrated seismic gathers as a part of the residual velocity analysis workflow.

\section{ACKNOWLEDGMENTS}

Figure 14. Stacked sections after (a) conventional NMO correction with $150 \%$ stretch mute and (b) MPNMO correction algorithm shown in Figure 3. The target PaleoceneEocene Chicontepec formation lies between $t=0.8 \mathrm{~s}$ and $t=1.2 \mathrm{~s}$ (Sarkar, 2011). Note the improved resolution (such as the events marked by the circle) and continuity (such as the event marked by the rectangle) of the reflection events using MPNMO correction method.

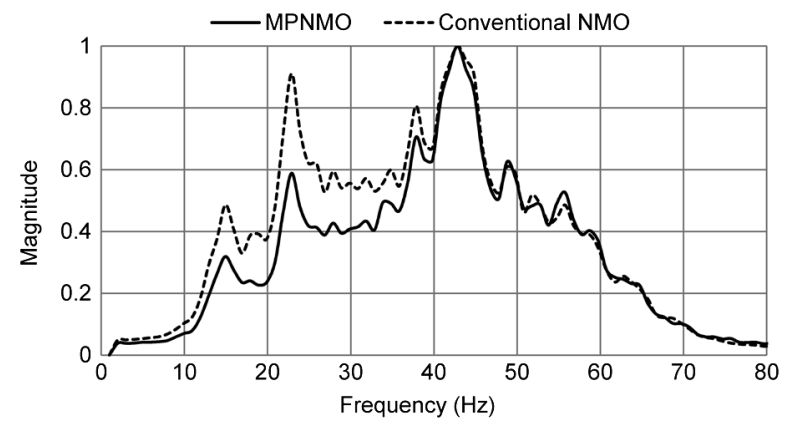

Figure 15. Average amplitude spectra for stacked sections shown in Figure 14 corresponding to the conventional NMO corrected gathers in dashed, and MPNMO-corrected gathers in solid curves. comments and suggestions of associated editor, Tariq Alkhalifah, reviewer Art Barnes, and two anonymous referees.

\section{REFERENCES}

Alkhalifah, T., 1997, Seismic data processing in vertically inhomogeneous TI media: Geophysics, 62, 662-675, doi: 10.1190/1.1444175.

Alkhalifah, T., 1998, Acoustic approximations for processing in transversely isotropic media: Geophysics, 63, 623-631, doi: 10.1190/1.1444361.

Barnes, A. E., 1992, Another look at NMO stretch: Geophysics, 57, 749-751, doi: $10.1190 / 1.1443289$.

Bolshykh, S. F., 1956, About an approximate representation of the reflected wave traveltime curve in the case of a multi-layered medium: Applied Geophysics (in Russian), 15, 3-15.

Brouwer, J. H., 2002, Improved NMO correction with a specific application to shallow seismic data: Geophysical Prospecting, 50, 225-237, doi: 10 $.1046 / \mathrm{j} .1365-2478.2002 .00310 . x$. 
Bruland, L. and T. A. Johansen, 1994, Resolving interfering events through cyclic sampling and median filtering: First Break, 12, 445-452.

Buchholtz, H., 1972, A note on signal distortion due to dynamic (NMO) corrections: Geophysical Prospecting, 20, 395-402, doi: 10.1111/gpr .1972.20.issue-2.

Byun, B. S., and E. S. Nelan, 1997, Method and system for correcting seismic traces for normal move-out stretch effects: U.S. Patent 5, 684, 754

Castle, R. J., 1994, A theory of normal moveout: Geophysics, 59, 983-999, doi: $10.1190 / 1.1443658$

de Bazelaire, E., 1988, Normal moveout revisited: Inhomogeneous media and curved interfaces: Geophysics, 53, 143-157, doi: 10.1190/1.1442449.

de Bazelaire, E., and J. R. Viallix, 1994, Normal moveout in focus: Geophysical Prospecting, 42, 477-499, doi: 10.1111/gpr.1994.42.issue-5.

Dix, C. H., 1955, Seismic velocities from surface measurements: Geophysics, 20, 68-86, doi: 10.1190/1.1438126.

Dunkin, J. W., and F. K. Levin, 1973, Effect of normal moveout on a seismic pulse: Geophysics, 38, 635-642, doi: 10.1190/1.1440363.

Fomel, S., and A. Stovas, 2010, Generalized nonhyperbolic moveout approximation: Geophysics, 75, no. 2, U9-U18, doi: 10.1190/1.3334323.

Hicks, G. J., 2001, Removing NMO stretch using the Radon and FourierRadon transforms: 63rd Annual Conference and Exhibition, EAGE, Extended Abstracts, A-18.

Hilterman, F., and C. Van Schuyver, 2003, Seismic wide-angle processing to avoid NMO stretch: 73rd Annual International Meeting, SEG, Expanded Abstracts, 215-218.

Hunt, L., S. Trickett, D. Levesque, P. McKenny, B. Link, and S. Jamieson, 2003 , The effect of stretch free stacking on a clastic exploration play in Alberta, Canada: 73rd Annual International Meeting, SEG, Expanded Abstracts, 321-324.

Key, S., and S. B. Smithson, 1990, New approach to seismic-reflection even detection and velocity determination: Geophysics, 55, 1057-1069, doi: 10 $.1190 / 1.1442918$.

Lichman, E., 1999, Automated phase-based moveout correction: 69th Annual International Meeting, SEG, Expanded Abstracts, 1150-1153.

Liu, J., and K. J. Marfurt, 2005, Matching pursuit decomposition using Morlet wavelet: 75th Annual International Meeting, SEG, Expanded Abstracts, 786-789.
Liu, J., and K. J. Marfurt, 2007, Instantaneous spectral attributes to detect channels: Geophysics, 72, no. 2, P23-P31, doi: 10.1190/1 .2428268 .

Masoomzadeh, H., P. J. Barton, and S. C. Singh, 2010, Nonstretch moveout correction of long-offset multichannel seismic data for subbasalt imaging: Example from the North Atlantic: Geophysics, 75, no. 4, R83-R91, doi: 10.1190/1.3443579.

Miller, R. D., 1992, Normal moveout stretch mute on shallow-reflection data: Geophysics, 57, 1502-1507, doi: 10.1190/1.1443217.

Noah, J. T., 1996, NMO stretch and subtle traps: The Leading Edge, 15, 345-347, doi: 10.1190/1.1437335.

Owusu, J. C., and T. W. Spencer, 1995, VSP normal moveout stretch: Geophysics, 60, 1579-1582, doi: 10.1190/1.1443891.

Perroud, H., and M. Tygel, 2004, Nonstretch NMO: Geophysics, 69, 599 607, doi: 10.1190/1.1707080.

Rupert, G. B., and J. H. Chun, 1975, The block move sum normal moveout correction: Geophysics, 40, 17-24, doi: 10.1190/1.1440511.

Sarkar, S., 2011, Depositional history and reservoir characteristics of structurally confined for deep turbidites, Northern Chicontepec Basin, Mexico: Ph.D. thesis The University of Oklahoma.

Shatilo, A., and F. Aminzadeh, 2000, Constant normal-moveout (CNMO) correction: A technique and test results: Geophysical Prospecting, 48, 473-488, doi: 10.1046/j.1365-2478.2000.00190.x.

Swan, H. W., 1988, Amplitude versus offset analysis in a finely layered media: 58th Annual International Meeting, SEG, Expanded Abstracts, 1195-1198.

Swan, H. W., 1997, Removal of offset-dependent tuning in AVO analysis: 67th Annual International Meeting, SEG, Expanded Abstracts, $175-178$.

Trickett, S., 2003, Stretch-free stacking: 73rd Annual International Meeting, SEG, Expanded Abstracts, 2008-2011.

Ursin, B., and B. O. Ekren, 1995, Robust AVO analysis: Geophysics, 60 317-326, doi: 10.1190/1.1443768.

Ursin, B., and A. Stovas, 2006, Traveltime approximations for a layered transversely isotropic medium: Geophysics, 71, no. 2, D23-D33, doi: $10.1190 / 1.2187716$. 